\author{
Submitted: \\ 18.06.2020 \\ Accepted: \\ 23.07.2020 \\ Published: \\ 28.09.2020

\section{POCUS in COVID-19: a dynamic tool during RSI} \\ Prakash Ranjan Mishra, Sanjeev Bhoi, Roshan Matthew, \\ Tej Prakash Sinha \\ Department of Emergency Medicine, All India Institute of Medical Sciences, New Delhi \\ Correspondence: Dr. Roshan Matthew, Senior Resident, Department of Emergency Medicine, \\ All India Institute of Medical Sciences, New Delhi,110029; tel.: +91-7838677063, \\ e-mail:roshmat15@gmail.com
}

DOI: $10.15557 /$ JoU.2020.0040

\begin{abstract}
Novel coronavirus (COVID-19) pandemic has been a concern for mankind and healthcare workers (HCW) for the past few months. About $5 \%$ of the patients become critically ill and require intubation and mechanical ventilation. Rapid sequence intubation (RSI) has been the cornerstone of airway management for decades. In today's era of COVID, applying and following the conventional principles of RSI may lead to significant exposure to viral load for HCW. Pre-oxygenation using bag-and-mask ventilation (BMV) is not a recommended practice in COVID suspect or positive patients ${ }^{(1)}$. It has an inherent risk of aerosol generation during the procedure, which may be hazardous ${ }^{(2)}$. Nasal cannulas, face masks or helmet oxygenation devices are relatively safe alternatives in these patients ${ }^{(1)}$.
\end{abstract}

During endotracheal intubation, the airway physician (AP) has to visualize the glottis for correct endotracheal tube (ETT) placement, under direct laryngoscopic vision. This may pose problems and challenges in current circumstances. Visualizing the glottis while using protective goggles and face shields is a tedious task because of the associated fogging and poor vision, a problem which most of us have encountered. Many centers use intubation box chambers and similar apparatuses to protect the AP during intubation, which adds to the challenge ${ }^{(3)}$. Simson JP et al. showed in their study that such devices may increase rather than decrease airborne particle exposure ${ }^{(4)}$. Video laryngoscopes, a necessity during these times, may not be available at all centers, especially in resource-constraint settings. This adds to the fear encountered by the emergency care providers while managing such patients, adversely affecting airway management and hence outcome of these patients.

The position of an endotracheal tube needs to be confirmed before airway management is declared to be complete. End-tidal $\mathrm{CO}_{2}\left(\mathrm{EtCO}_{2}\right)$, the gold standard for correct confirmation of endotracheal tube, may not be available at all centers. Five-point auscultation, a practice at most centres, has a sensitivity of $94 \%$ and specificity $83 \%{ }^{(5)}$ and carries the inherent risk of aerosol generation, and hence disease transmission. It also requires at least four-five AMBU breaths before a reading displayed can be relied upon $^{(5)}$. Auscultation of a patient to rule out right mainstem bronchus intubation is very difficult for a physician with full personal protective equipment (PPE), with safety hoods covering the head and ears.

This may be especially more challenging in Emergency room settings. Point-of-care ultrasound (POCUS) can be a handy tool in this current situation. Bhoi et al. have described the role of POCUS in COVID19 pandemic $^{(6)}$.

The authors feel that POCUS can be used effectively during RSI to address the concerns associated with conventional methods of intubation and tube confirmation during RSI in COVID-19 patients (Fig. 1). Ultrasound can be used initially to locate the esophagus and access the airway anatomy in order to rule out a difficult airway. Hall et al. in their feasibility study of using ultrasound to assess airway found a good correlation with Mallampatti score ${ }^{(7)}$. This can help in better planning and preparation in cases of a failed attempt to intubate. Then using a linear US probe (7-12 MHz) kept transversely over the neck, we can visualize the passage of ETT through the glottis and trachea. Dynamic visualization of widening of glottis as the ETT passes through, clearly visible posterior wall of the esophagus seen post-intubation (seen as a bright hyperechoic line) and absence of 'doubletract sign' confirm endotracheal intubation and excludes entry of ETT in the false lumen, i.e. esophagus (Fig. 2). Exclusion of esophageal intubation can be done in realtime, hence obviating the need for re-intubation after fivepoint auscultation or after reading $\mathrm{EtCO}_{2}$ values $^{(8)}$. During 


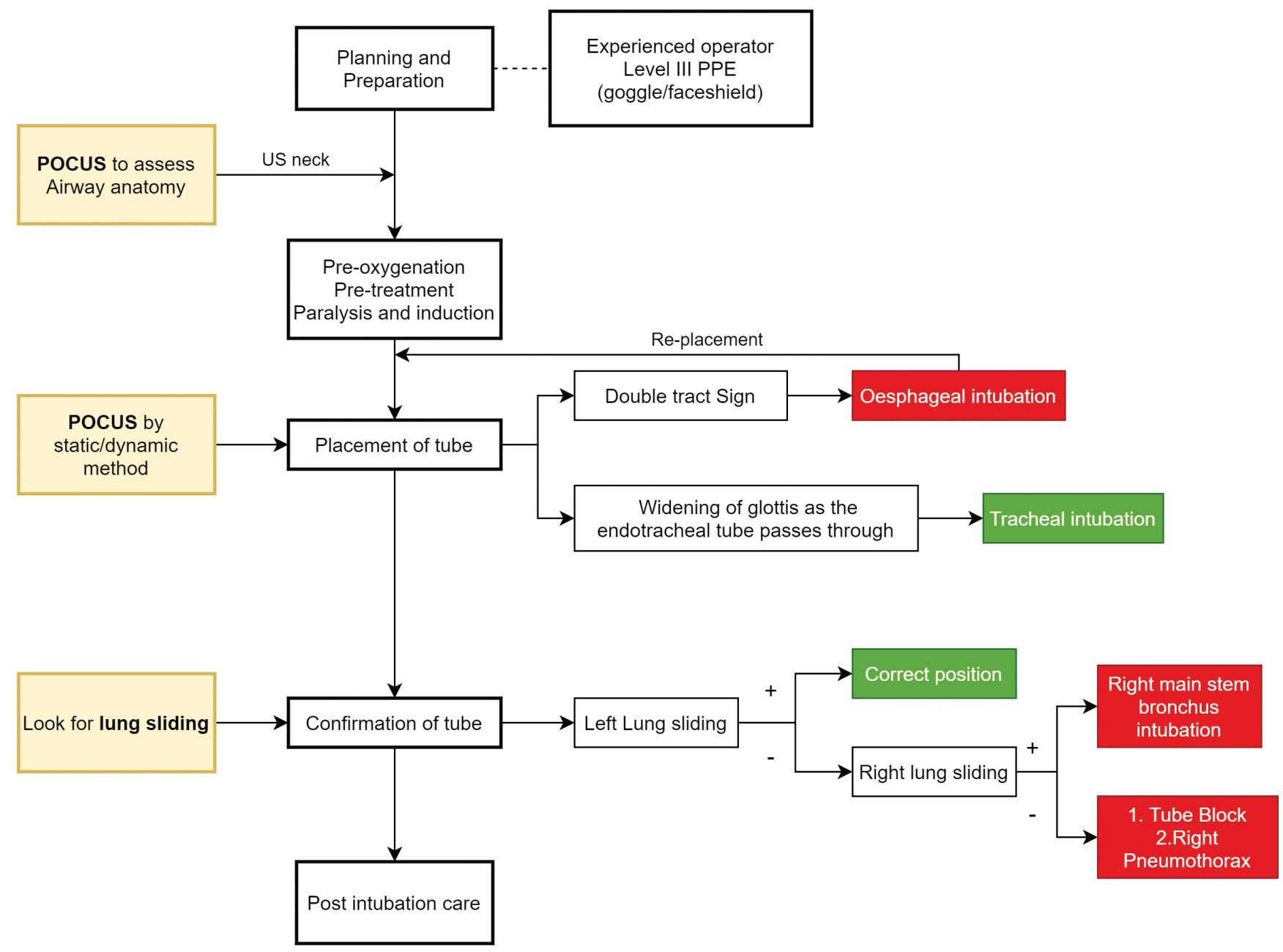

Fig. 1. Integration of POCUS with Rapid Sequence Intubation

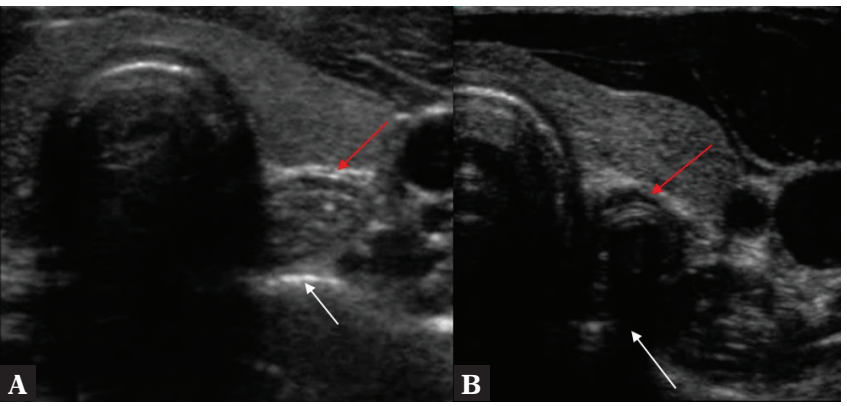

Fig. 2. A. Transverse view of neck above the suprasternal notch at the level of thyroid showing the esophagus (red arrow). The posterior wall is seen clearly as a bright hyperechoic line (white arrow). B. Transverse view of the neck above the suprasternal notch at the level of thyroid showing esophageal intubation. the presence of a 'double-tract sign' (red arrow) and invisible posterior wall of the esophagus (white arrow head) confirms esophageal intubation

the post-intubation phase, POCUS is helpful to confirm that the location of ETT is correct, i.e. above the carina. the presence of left lung sliding during lung scan virtually rules out right mainstem bronchus intubation. The right subcostal view to look for diaphragm movement is found to be sensitive and specific secondary confirmatory method to check correct ETT placement ${ }^{(9)}$.

Hence, POCUS during RSI ensures that all the inherent problems and risks associated with conventional methods of intubation and tube confirmation are minimized or completely avoided. In order to minimize cross infection, a separate ultrasound machine should be designated with probe covers and low-level disinfectants can be used ${ }^{(4)}$. Integrating POCUS with RSI will boost confidence in HCW, decrease the strain on available health-care resources and also help in preserving the healthy work-force of hospitals.

Hence, the authors advocate and recommend the use of POCUS during RSI in all patients with suspected or confirmed coronavirus disease, for whom definitive airway management is planned.

\section{Conflict of interest}

Authors do not report any financial or personal connections with other persons or organizations, which might negatively affect the contents of this publication and/or claim authorship rights to this publication. 


\section{References}

1. Cheung JC-H, Ho LT, Cheng JV, Cham EYK, Lam KN: Staff safety during emergency airway management for COVID-19 in Hong Kong. Lancet Respir Med 2020; 8: e19. https://doi.org/10.1016/S22132600(20)30084-9.

2. El-Boghdadly K, Wong DJN, Owen R, Neuman MD, Pocock S, Carlisle JB, et al.: Risks to healthcare workers following tracheal intubation of patients with COVID-19: a prospective international multicentre cohort study. Anaesthesia 2020. https://doi.org/10.1111/anae.15170.

3. Cook TM, El-Boghdadly K, McGuire B, McNarry AF, Patel A, Higgs A: Consensus guidelines for managing the airway in patients with COVID-19. Anaesthesia 2020; 75: 785-799. https://doi.org/10.1111/ anae. 15054.

4. Simpson JP, Wong DN, Verco L, Carter R, Dzidowski M, Chan PY: Measurement of airborne particle exposure during simulated tracheal intubation using various proposed aerosol containment devices during the COVID-19 pandemic. Anaesthesia 2020. https://doi.org/10.1111/ anae. 15188 .
5. Grmec S: Comparison of three different methods to confirm tracheal tube placement in emergency intubation. Intensive Care Med 2002; 28: 701-704. https://doi.org/10.1007/s00134-002-1290-x.

6. Bhoi S, Sahu A, Mathew R, Sinha T: Point-of-care ultrasound in COVID-19 pandemic. Postgrad Med J 2020. https://doi.org/10.1136/postgradmedj-2020-137853.

7. Hall EA, Showaihi I, Shofer FS, Panebianco NL, Dean AJ: Ultrasound evaluation of the airway in the ED: a feasibility study. Crit Ultrasound J 2018; 10: 3. https://doi.org/10.1186/s13089-018-0083-6.

8. Mishra PR, Bhoi S, Sinha TP: Integration of point-of-care ultrasound during rapid sequence intubation in trauma resuscitation. J Emerg Trauma Shock 2018; 11: 92-97. https://doi.org/10.4103/JETS. JETS_56_17.

9. Hosseini JS, Talebian MT, Ghafari MH, Eslami V: Secondary confirmation of endotracheal tube position by diaphragm motion in right subcostal ultrasound view. Int J Crit Illn Inj Sci 2013; 3: 113-117. https:// doi.org/10.4103/2229-5151.114269. 